Shaoze Zhang

Xianbo Xiang

Shaolong Yang

http://dx.doi.org/10.21278/brod71402

ISSN 0007-215X

eISSN 1845-5859

\title{
COMMUNICATION NETWORK AND QOS EVALUATION FOR FORMATION CONTROL OF UNMANNED SURFACE VEHICLES
}

UDC 629.5.066.3

Original scientific paper

\begin{abstract}
Summary
The main purpose of this article is to design efficient communication networks for the formation control of multiple distributed Unmanned Surface Vehicles (USVs). First, a hybrid communication network architecture is proposed by combining remote communication and wireless Ad hoc network technology. Second, an improved Low Energy Adaptive Clustering Hierarchy $(\mathrm{LEACH})$ protocol is adopted to prolong the life cycle of the communication network of the USV fleet. Subsequently, some Quality of Service (QoS) indicators of the USV communication network are evaluated by establishing wireless network channel model and Signal to Interference and Noise Ratio (SINR) model. In particular, the packet error ratio, average time delay and connectivity under different formation architecture are investigated. Finally, some discussions and future work on the QoS of the USV communication network are concluded.
\end{abstract}

Key words: multiple USVs; Ad hoc network; LEACH clustering algorithm; Communication network architecture; Quality of Service $(Q o S)$

\section{Introduction}

With the development of intelligent technologies, the use of advanced marine equipment and systems [1-6], has been increased rapidly to accomplish missions in complex marine environments. As multi-agent's formation is capable of accomplishing multiple tasks as compared to single agent with enhanced work efficiency [7-9], multi-unmanned surface vehicles (multi-USVs) formation can be applied in search and rescue, patrol and marine transportation. Formation control of multi-USVs is the basis of above application. Accordingly, for effective implementation of the multi-agent system, its formation control is considered to be the most challenging and meaningful domain. Various formation control strategies are available such as virtual piloting, leader-followers, behaviour-based, and graphbased [10]. However, the design and implementation of formation controller is based on the guaranteed communication networks. During the formation control of multi-USVs, inaccurate positions will be used if the positions cannot be updated timely, which is adverse against the formation control. USVs network techniques have significant meanings for USVs formation control. Therefore, in order to guarantee the real-time formation control of Unmanned Surface 
Vehicles, Communication architecture, communication networks algorithm and quality of service (QoS) problem should be investigated.

USV communication network has similar characteristics to mobile WSN (Wireless Sensor Network) and other multi-agent's communication networks such as UAV (Unmanned Aerial Vehicle) communication networks. USV communication networks have the characteristics of mobility, limited energy and numerous nodes. In aspect of prior work of communication architecture design and evaluation of QoS, Mike et al. [11] used OPNET communication network simulation software to establish a model of the ship's combat system data network and model the application layer information flow, node interface and node behaviour of each node to realize the information interaction function of the entire combat network. Network performance such as network delay, flow ratio, link utilization ratio, was obtained through simulation of the flow information of combat system data network. Haque et al. [12] explored the issues of UAV communication networks, analyzed its characteristics, applications, routing protocols, quality of service, power issue and so on. Vishal et al. [13] focused on implementation of UAV (unmanned aerial vehicles) ad hoc network that forms a guidance system for ground ad hoc network. They used neural network to form cognitive and topology maps. It can be concluded that network techniques of UAVs have been researched including cooperative network framework, energy consumption, and QoS, Whereas, Research about USVs formation communication networks especially about evaluation of QoS indicators is not very rich.

As for the state of art about communication networks algorithm, Zhou et al. [14] adopted the Enhanced-Channel-Aware Routing Protocol (E-CARP) to create the development of Internet of Underwater Things. The principle objective was the achievement of an inexpensive data forwarding and less energy consumption system. Qiu et al. [15] proposed a routing protocol called Global Information Decision (ERGID) for energy response and delay estimation. The simulation result associated with time-delay, packet loss, and consumption of energy was considered in this research. Qiu et al. [16] also proposed a Greedy Model with Small World model (GMSW) to maintain the robustness of the IoT (Internet of things) structure with increased performance. they considered that the feasibility of the optimization algorithm was obtained by the small world model. It can be seen that researchers did some research and verification in the field of communication networks algorithm about Internet of things and underwater things for the issues of energy consumption and efficient data interaction.

About the formation network techniques, Pham et al [17] studied the formation control problem in a clustered network system. In each cluster, a node called a leader can communicate with other leaders outside the cluster at specific moments. Besides, the continuous-time communication topology in each cluster was represented by fixed and undirected graphs. The authors also did some prior work about USVs formation network. The designed formation controller based on leader-followers strategy for multi-USVs, and field experiment with four vehicles holding diamond structure formation were presented in [18]. ZigBee mesh network is adopted for USV communications. However, this method has some limitation, such as short communication range, finite number of router nodes. These may limit USVs formation scale and communication efficiency. Peng et al. [19] investigated distributed adaptive fault-tolerant control schemes for spacecraft formation subject to external disturbances, model uncertainties and communication delays. The simulated formation control problem was performed with a given time-delay. Although the influence of communication delay on formation control was considered, reasonably evaluating communication time delay of formation was not included. While considering the communication networks techniques for USVs, Namgung et al. [20] designed the communication relay system to enable navigation information exchange in a dedicated 
communication network via the Maritime Control Station and conducted experiment to verify the system. However, the formation control scenario was not combined with the communication network problem.

Based on the above investigation, this study therefore proposes a method to address the problem of communication between numerous USVs widely distributed and evaluate QoS indicators for Multi-USVs formation. As for unmanned surface vehicles that can operate in rivers, lakes, inland and offshore, A hybrid communication network architecture is proposed. It can realize the communication networks of unmanned surface vessels without geographical restrictions. Moreover, an improved cluster algorithm based on Low Energy Adaptive Clustering Hierarchy (LEACH) protocol is proposed, which can prolong life cycle of network. Furthermore, multi-USVs formation communication network model is designed, and method of evaluating QoS indicators is proposed.

The rest of the paper is organized as follows. In section 2, a wireless network hardware composition and the architecture design for numerous USV nodes are introduced. The main objective is to ensure that USV nodes can exchange data in hardware. In section 3, a cluster routing algorithm is proposed to design an efficient and reliable routine algorithm to manage the communication networks strategy and routing strategy between numerous USV nodes. In section 4, a network channel model and Signal to Interference and Noise Ratio (SINR) model are proposed. To evaluate QoS indicators of USVs' formation, the packet error ratio and delay model of the USV formation communication network is also proposed. In section 5, the simulation experiment background and method are introduced, and simulation results are discussed as well. The final section concludes the research and envisions the future work.

\section{Network Architecture Design for Multi-USVs}

In order to address the challenges of the short distance communication between USVs in hardware architecture level, four DRF1605H ZigBee module based on IEEE 802 standard are used to verify USVs formation network and field experiments are carried out [18]. ZigBee network is composed of coordinator, router, and device. The Zigbee coordinator is responsible for creating and maintaining the network [21]. Coordinator can transparently transmit data to all router node in ZigBee network, and all router can transmit data to other node in network by point to point supported by ZigBee protocol. As for WIFI protocol, WIFI network is tested by using TL-WN722N module. It can be tested ZigBee/WIFI protocol can build a LAN (Local Area Network) for USVs communication. However, ZigBee/WIFI and any other Ad hoc network protocol cannot meet the communication requirement of numerous and widely distributed USVs formation. To deal with this issue, 4G-DTU module are adopted for USVs formation. Using 4G communication technology, it can realize remote communication between USV and cloud server. While, 4/5G communication has high energy consumption and high cost. This method is suitable for remote control of single USV, but not for multi-USVs formation. Hence, a hardware architecture is proposed to deal with the communication requirement for USVs formation, which combines the Ad hoc network and remote communication network, as shown in Fig. 1.

In each USVs formation group, USVs within a specific communication range adopt Ad hoc network for data interaction to form a USVs group. There may be several USVs groups in a certain specific area. Each group has a base station node that can realize the communication between USV groups through remote communication network supported by $4 / 5 \mathrm{G}$ technology. Ad hoc network within a group can adopt various protocols such as ZigBee/WIFI. Simultaneously, the data of all USVs in the group can be collected and uploaded to the $4 / 5 \mathrm{G}$ module and the data interaction between USVs groups can be realized through cloud server. The proposed hardware architecture can be applied to realize numerous and widely distributed USVs communication. This architecture can achieve large-scale, wide-range and mobile 
USVs communication networks in inland rivers, lakes, and other scenarios to achieve efficient and reliable data interaction.

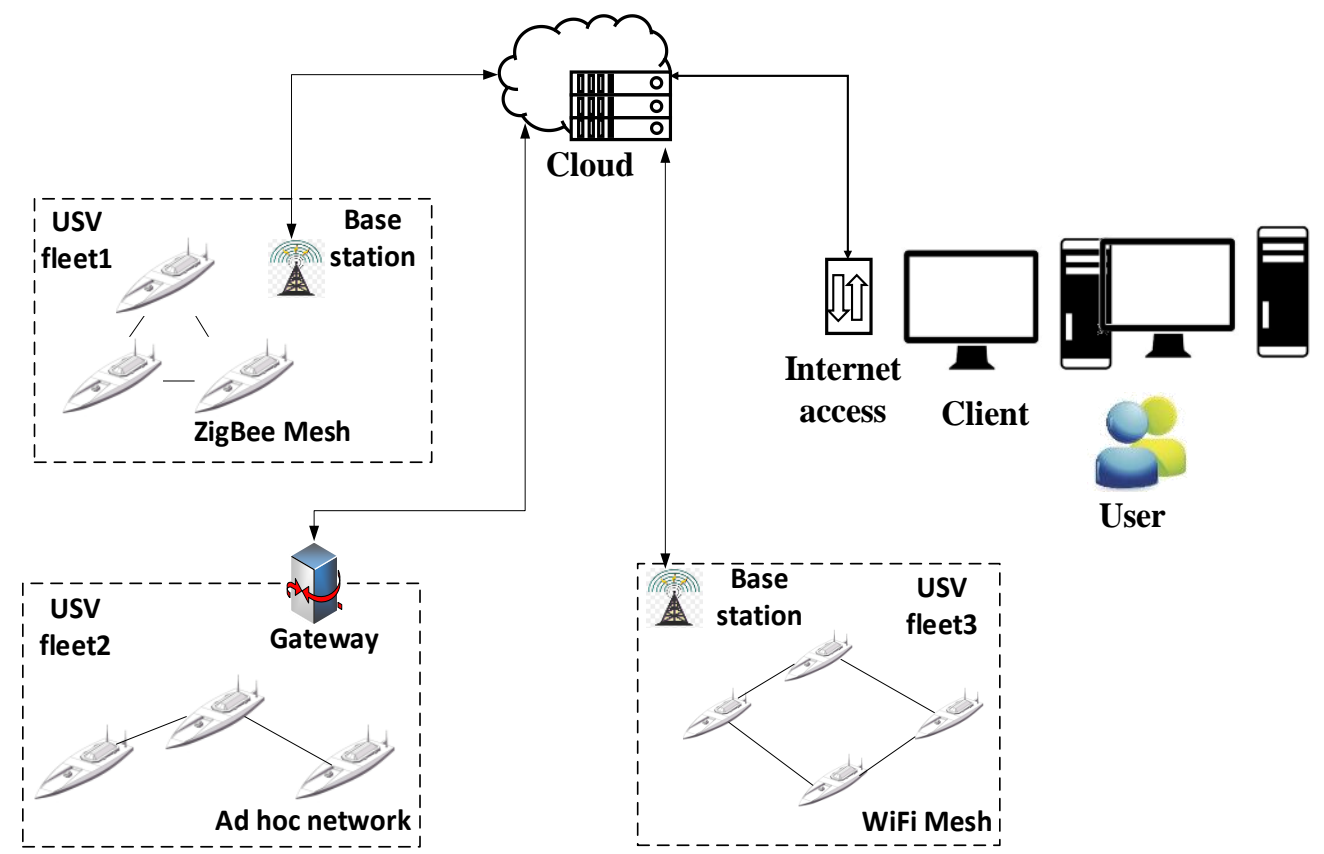

Fig. 1 Hardware architecture combining remote communication and ad hoc network for USV formation system

As a supplement, in the offshore navigation, without the support of the $4 / 5 \mathrm{G}$ mobile communication base station, the architecture of combining satellite communication and WIMAX network communication is proposed to realize the communication between manned/unmanned surface vessels. The vessels in a specific sea area can form a large communication packet through WIMAX to form a wireless ad hoc network. The communication packets can realize data interaction through maritime mobile buoys or satellite communications. The communication network architecture is shown in Fig. 2.

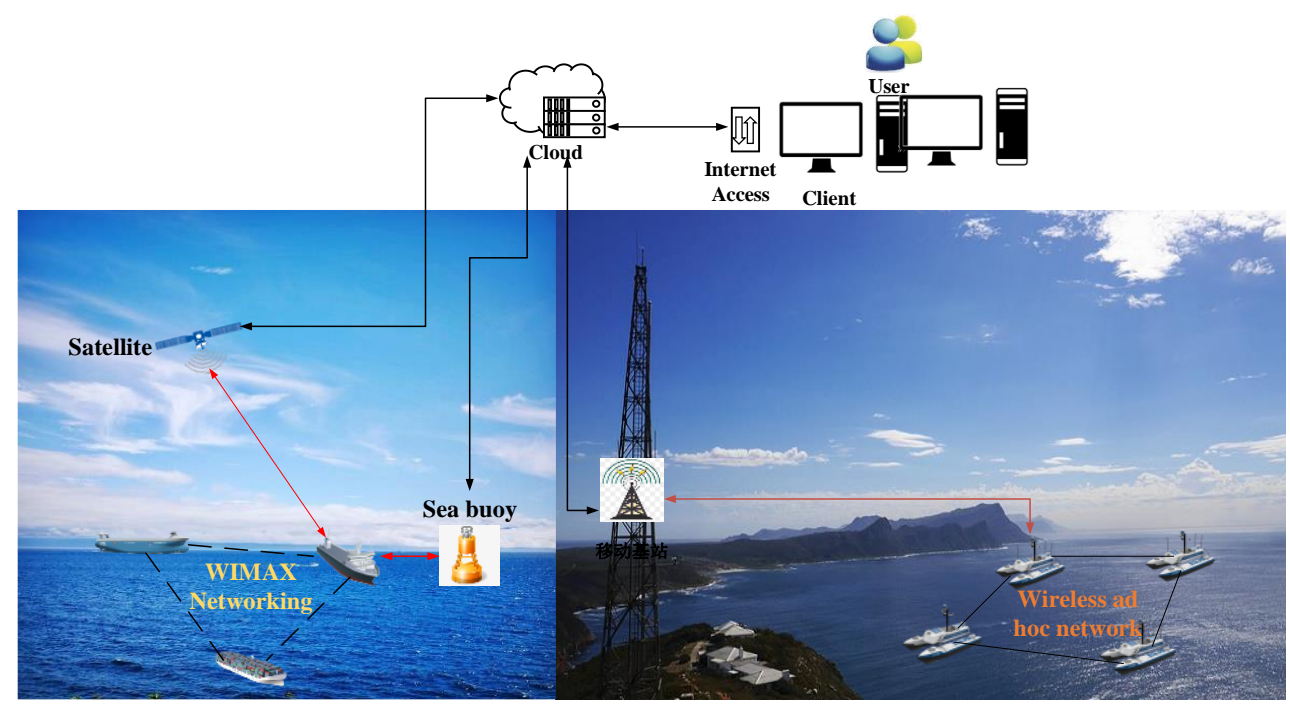

Fig. 2 Network architecture for inland/offshore onboard communication

The proposed communication network hardware architecture is termed as hybrid USVs communication network architecture which has a wide range of application. It combines the advantages of long-distance mobile communication technology and wireless ad hoc network technology to achieve remote, safe and efficient surface vessels network communication. Hybrid communication network architecture's characteristics are summarized in Table 1. 
Table 1: Hybrid communication architecture summary

\begin{tabular}{|c|c|c|c|c|}
\hline Architecture & Distance & Security & Mobility & Cost \\
\hline $\begin{array}{c}\text { 4/5G plus } \\
\text { Ad hoc }\end{array}$ & $\begin{array}{c}4 / 5 \mathrm{G} \text { signal } \\
\text { coverage area }\end{array}$ & $\begin{array}{c}\text { High } \\
\text { security }\end{array}$ & $\begin{array}{c}\text { Support } \\
\text { mobility }\end{array}$ & Low cost \\
\hline $\begin{array}{c}\text { Satellite plus } \\
\text { WIMAX }\end{array}$ & Unlimited & $\begin{array}{c}\text { High } \\
\text { security }\end{array}$ & $\begin{array}{c}\text { Support } \\
\text { mobility }\end{array}$ & High cost \\
\hline
\end{tabular}

\section{Communication Networks Algorithm for Multi-USVs Formation}

The USV formation system can exchange data between any USV nodes through the communication network architecture described in section 2. Whereas, it is necessary to consider USV communication networks have limited capability in terms of power of battery, bandwidth and processing, and numerous nodes transfer multiple data from node to the base station about the same event, which leads to the transfer of redundant data [15], and this may cause huge data throughput of the entire network, and frequent data exchanges between nodes may bring a lot of energy consumption and may also cause blocking/congestion of the entire communication networks resulting in an increase of delay and decline in the quality of service. Therefore, it is necessary to design a communication networks strategy to address the problem of deciding which nodes should communicate mutually, that is, routing algorithm problem in communication networks.

Node clustering is an improved method to improve the network scalability and life time [22]. Considering the algorithm's complexity and scalabilities such as low network delay, load balancing, low algorithm complexity, and easy implementation, we adopted the LEACH algorithm for the networking of USVs' formation system. The LEACH algorithm can be divided into three stages that are cluster head node election, cluster form through joining the head nodes, and data transmission. This process runs periodically. The nodes participating in the competition at the first stage of the election will be randomly selected to become a cluster head with a certain probability. Nodes that can become the cluster head before the start of each round will participate in the competition, generate a random number and compare the random number with the node's threshold. The greater the threshold of a node, the higher the probability as it can be selected as a cluster head.

In the LEACH algorithm, the threshold value can be computed using Equation (1) [23]:

$$
T(n)= \begin{cases}\frac{p}{1-p\left(r \bmod \frac{1}{p}\right)} & , n \in G \\ 0 & \text { otherwise }\end{cases}
$$

Where $n$ is the node number, $p$ is the expected cluster head ratio, $r$ is the round and $G$ is the set of nodes which can be selected as the cluster head.

After the election of the head node of the cluster, remaining ordinary nodes join the cluster head nodes closest to them to form clusters. In the data transmission stage, the cluster head interacts with each cluster member node. Each cluster head node interacts through the base station node, which can ensure that each node in the USVs' formation obtains sufficient information for formation control. Therefore, the USVs network can use the LEACH algorithm to establish and maintain data transmission paths.

LEACH algorithm applied to the USVs formation can solve the problem of large-scale node networking in the USVs' formation system. At the same time, this study has also made 
improvements based on the LEACH algorithm in the communication networks algorithm. In the cluster head election process, three factors were taken into consideration: candidate cluster head neighbour nodes, candidate node residual energy and distance between the candidate node and base station. Accordingly, the threshold $T(n)$ is modified and can be computed using equation (2).

$$
T^{\prime}(n)= \begin{cases}\frac{W * p}{1-p\left(r \bmod \frac{1}{p}\right)}, & n \in G \\ 0 & \text { otherwise }\end{cases}
$$

The correction factor $W$ is defined as formula (3):

$$
W=A * \frac{E_{i}(r)}{E_{\max }(r)}+B * \frac{D(i)}{D_{\max }}+C * \frac{d_{a v g}}{d(i)}
$$

Where $A, B, C$ are the node residual energy, node degree and distance from the node to base station control factor, respectively. $E_{i}(r)$ represents the node residual energy and $E_{\max }(r)$ represents the maximum value of the node's remaining energy in the r-round system. $D(i)$ represents the number of neighbours and $D_{\max }$ represents the maximum number of neighbours. $d(i)$ represents the distance from the node to the base station and $d_{a v g}$ represents the average distance from the node to the base station.

By changing the control factors $A, B, C$, the system can improve the network life cycle, balance the network load, reduce the delay of cluster head nodes and improve the networking efficiency of the USVs' formation. There is a detailed description of selecting control parameters of $A, B, C$ in section 5.2.

\section{Communication Networks Model and QoS Evaluation Method}

Multi-agent's formation control is sensitive to QoS indicators such as time delay and packet error ratio of the communication networks. Taking the leader-followers formation control strategy as an example, the formation shape may fail to maintain due to failure of updating the pilot's position and other information within a certain period of time. Similarly, the key to AGV (Autonomous Ground Vehicle) formation is lane-keeping and early warning of collisions like the car's position is constrained by the lane. The focus is usually on realtime communication to continuously update the information between to maintain the formation shape. UAV (Unmanned Aerial Vehicle) networking is mainly used in numerous UAV collaboration, the position is highly constrained in three-dimensional space, and the position change is limited. However, USVs usually work in a wide area of lakes or seas, and their position constraints are relatively loose. Therefore, on the premise of meeting the realtime requirements in formation control, evaluating the QoS indicators can provide a basis for maintaining the formation shape and optimizing the formation efficiency and communication quality of service.

In order to evaluate the QoS indicators for USVs' formation communication networks, the main indicators to be evaluated are packet error ratio, end-to-end network delay and node connectivity ratio. Assuming that there is no network blockade/congestion (excluding the queuing delay) and ignoring the processing delay, the end-to-end network delay can be expressed as equation (4): 


$$
\tau_{\text {end-to-end }}=N\left(\text { delay }_{\text {trans }}+\text { delay }_{\text {prop }}\right)
$$

Where, $N$ is the number of hops, passed by end-to-end communication and delay $y_{\text {trans }}$ is the network transmission delay and can be computed using expression (5). Similarly, delay $y_{\text {prop }}$ is the propagation delay of the network and can be computed using expression (6):

$$
\begin{aligned}
& \text { delay }_{\text {trans }}=\frac{L}{R} \\
& \text { delay }_{\text {prop }}=\frac{M}{c}
\end{aligned}
$$

where $L$ is the packet length, and $R$ is the network transmission ratio $M$ is the propagation medium's length, and $c$ is the transmission ratio of electromagnetic waves in the medium.

To evaluate the end-to-end communication networks time delay of the USVs' formation, it is essential to identify the environmental noise and the interference of the nodes of other USVs in the network. Both of these factors are to be considered with actual physical network model. USVs located on the water surface can be regarded as operating at the same level with respect to vertical altitude. Therefore, its communication channel quality depends on the distance between USVs. Accordingly, the model is simplified by assuming that the communication link between USVs is Line of Sight (LOS) model.

The channel power gain from USV $i$ to $j$ in a certain period of time follows the freespace path loss model, which can be expressed as equation (7):

$$
h_{i j}=\frac{\rho_{0}}{\left\|\mathrm{q}_{i}-q_{j}\right\|^{2}}=\frac{\rho_{0}}{d_{i j}^{2}}
$$

where $\rho_{0}$ is the unit distance channel gain, $d_{i j}^{2}$ is the distance between USV $i$ and $j$.

When the USV node $i$ transmits data to node $j$, the signal-to-interference and noise ratio SINR can be expressed as equation (8) [24]:

$$
\operatorname{SINR}=\frac{h_{i j} P_{i}}{I_{i j}+N}=\frac{h_{i j} P_{i}}{\sum_{k \in I} h_{k j} P_{k}+\sigma^{2}}
$$

where $P$ represents the power of the USV node to send data, and $\sigma^{2}$ is the communication environment noise. $\sum_{k \in I} h_{k j} P_{k}$ represents interference items, and set $I$ represents the set of nodes that cause interference during sending data.

In the above expression, it is difficult to estimate the set of nodes I in the communication networks process. However, it can be obtained using probability estimation techniques, assuming that the Media Access Control (MAC) layer of the communication device in the USVs' nodes follow the Time Division Multiple Access (TDMA) protocols. This allows multiple nodes to use frequency resources simultaneously in different time intervals. 
Supposing nodes randomly send data, independent of each other, with probability $r$, The expected interference in the process of data transmission from node $i$ to node $j$ can be estimated using expression (9).

$$
\sum_{k \in N, k \neq i, j} r h_{k j} P_{k}
$$

The signal-to-interference and noise ratio can be expressed as Equation (10).

$$
\operatorname{SINR} \approx \frac{h_{i j} P_{i}}{\sum_{k \in N, k \neq i, j} r h_{k j} P_{k}+\sigma^{2}}
$$

To obtain the result in $d B$, Equation (10) can be written as.

$$
\gamma_{i j}=10 \lg \frac{h_{i j} P_{i}}{\sum_{k \in N, k \neq i, j} r h_{k j} P_{k}+\sigma^{2}}
$$

The packet error ratio can be estimated expression (12). Where, the value of $a_{n}, g_{n}$ depends on the hardware conditions in that SINR and noise ratio of packet error ratio is less than one [25].

$$
\operatorname{PER}\left(\gamma_{i j}\right)= \begin{cases}1 & \gamma_{i j}<\gamma_{p n} \\ a_{n} \exp \left(-g_{n} \gamma_{i j}\right) & \gamma_{i j} \geq \gamma_{p n}\end{cases}
$$

Finally, the end-to-end network delay under signal interference and environmental noise conditions can be obtained as formula (13).

$$
\alpha_{i j}=\frac{\tau_{\text {end-to-end }}}{1-\gamma_{i j}}
$$

Hence, USVs formation communication networks algorithm is developed. The evaluation of the QoS indicators for the packet error ratio, end-to-end network delay and node connectivity ratio is achieved obtained. QoS indicators can be calculated during data transmission based on Line of Sight communication model. USVs formation communication networks algorithm and QoS evaluation method is shown in Fig. 4. 


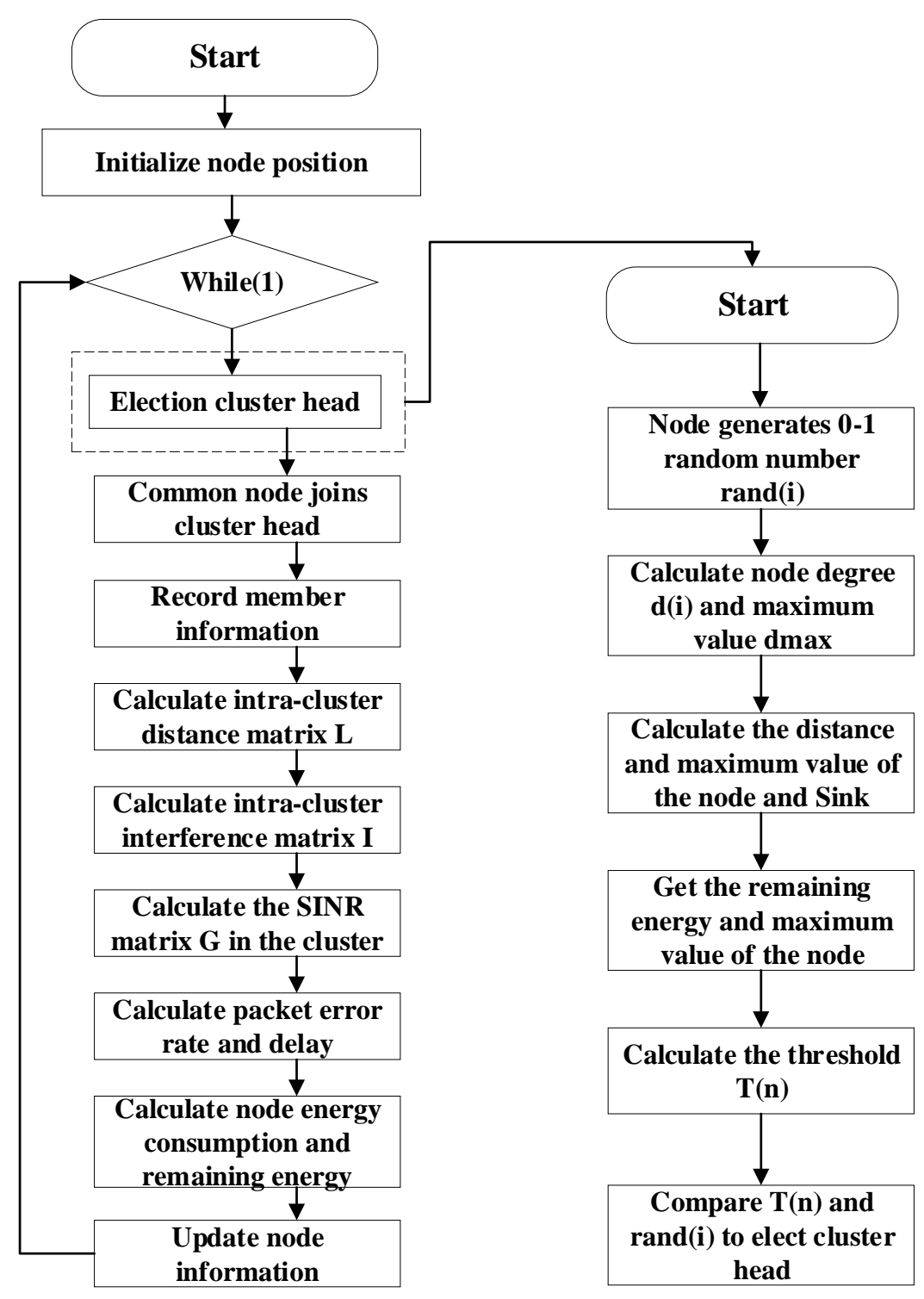

Fig. 4 USVs formation network and QoS estimation algorithm flow chart

The description of algorithm in flowchart as follows:

Step 1: Generate nodes according to formation position and assign random number, and set type as 'N'.

Step 2: Compare the random values of all nodes with type ' $N$ '.

Step 3: If random number is greater than the threshold value, the cluster head is selected and set to ' $\mathrm{C}$ '.

Step 4: Calculate the Euclidean distance between common nodes and all cluster heads to form the distance matrix.

Step 5: Using min function to find the cluster head nearest to the normal node and connect them.

Step 6: Calculate the interference matrix, SINR matrix, QoS indicators and energy consumption and record these parameters.

Step 7: Return to Step 1 to start the next round of clustering. 


\section{Simulation Verification}

\subsection{Simulation setup}

To verify the effectiveness of the USVs' formation network algorithm and to analyze the QoS indicators, numerical simulations are carried out under the simulated field experiments. First, several USVs need to be equipped, Second, suitable communication module needs to be obtained to realize the function of physical layer and data link layer and open source routing algorithm layer for modification. Third, communication test tool needs to be equipped to verify the accuracy and scientific of evaluation results. By considering the constraints of the conditions described above, the simulation experiments are designed as follows.

1) About the scenario and model, USV is taken as a point distributed in the range of $100 \mathrm{~m} * 100 \mathrm{~m}$ with a certain initial energy. The communication model adopts LOS (line of sight) link [26].

2) About the implementation of simulations, the simulation program runs in a cycle, and each cycle will drive the LEACH algorithm and calculate energy consumption and QoS indicators shown as the flowchart in Fig. 4. Detailed configuration about the simulation experiment is shown in Table 2.

Table 2: Detailing simulation and experiment design parameter settings

\begin{tabular}{|c|c|c|c|c|c|}
\hline Platform & Method & USV model & $\begin{array}{c}\text { Communication } \\
\text { model }\end{array}$ & Range & Round \\
\hline MATLAB & Custom code & $\begin{array}{c}\text { Point with } \\
\text { energy }\end{array}$ & LOS link & $100 \mathrm{~m} * 100 \mathrm{~m}$ & 1200 \\
\hline
\end{tabular}

\subsection{Simulation results for improved LEACH algorithm}

In order to check the improved LEACH algorithm, 100 random nodes and energy control parameters are chosen as $\mathrm{A}=0.3, \mathrm{~B}=0.2, \mathrm{C}=0.5$. $\mathrm{A}, \mathrm{B}, \mathrm{C}$ parameters represent different energy factor terms. The larger the parameter, the greater the impact of this energy factor on the communication energy consumption. When the number of nodes is large, the impact of communication energy consumption of base station may be lower than that of members. In this case, parameters A and B should be increased. According to different scenarios and energy consumption requirements, different parameters can be combined to optimize energy consumption.

The simulation results record the number of surviving nodes, the average value of the node's remaining energy and other indicators during the networking process. Comparison of improved LEACH with correction factors and traditional LEACH algorithm is shown in Fig. 5 . 


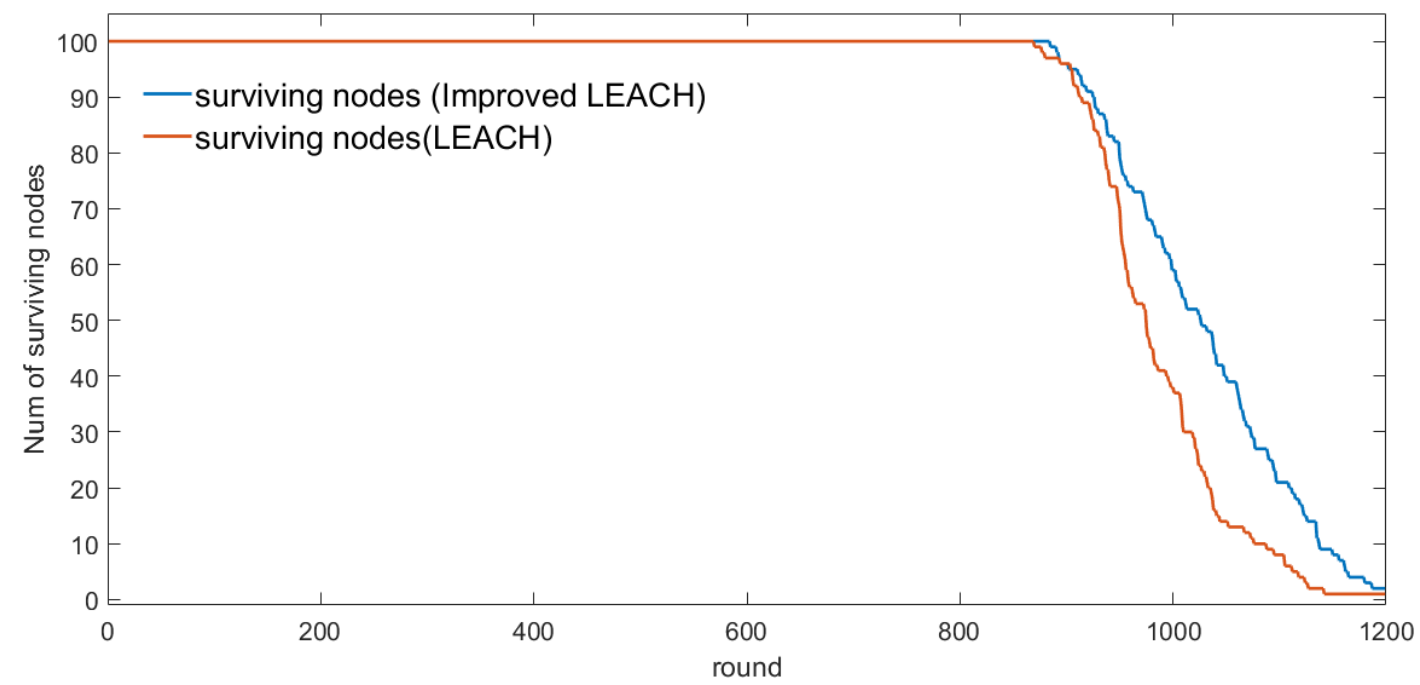

Fig. 5 Comparison of changes in the number of surviving nodes of USVs

The horizontal axis in Fig. 5 represents the communication networks rounds of the USVs' formation nodes. Each communication round can be a certain multiple of USVs' formation control cycle. After each round, the USV nodes will re-elect cluster head according to the clustering algorithm and regenerate network topology. By comparing the simulation results in Fig. 5, it is observed that the LEACH clustering algorithm with correction factor can save node energy, balance network load and improve network life cycle as compared to the traditional LEACH algorithm in the process of large-scale node networking communication. By quantifying the simulation results, it shows that after the 1200th round, the node survival ratio of the improved $\mathrm{LEACH}$ algorithm is $59 \%$ whereas as the same is $38 \%$ for the traditional algorithm.

\subsection{Simulation results for evaluating QoS indicators of USVs formation}

QoS indicators in USVs formation network are evaluated through simulation for two different scenarios. In the first case, geometric configuration of the USVs formation is different with a certain number of USV nodes. In the second case, the shape of the USVs' formation is fixed as diamond and the number of USV nodes is variable. The QoS index is evaluated and analysed as follows.

\subsubsection{QoS evaluation for different formation structure}

In the actual application scenario, USVs formation is usually need to be maintained during surface operations. Diamond formation is suitable for encirclement, triangle formation is more suitable for patrol, and column formation is mainly used for passing through narrow waters and avoiding obstacles. Simulation evaluation for above different formation shape are carried out. Parameter settings for the given case are listed in Table 3.

Table 3: Parameters under different formation geometry shape

\begin{tabular}{|c|c|c|c|c|c|}
\hline $\begin{array}{c}\text { Number of } \\
\text { nodes }\end{array}$ & $\begin{array}{c}\text { Formation } \\
\text { shape }\end{array}$ & $\begin{array}{c}\text { Unit channel } \\
\text { gain }\end{array}$ & $\begin{array}{c}\text { Environmental } \\
\text { noise }\end{array}$ & $\begin{array}{c}\text { Interference } \\
\text { ratio }\end{array}$ & $\begin{array}{c}\text { Transmission } \\
\text { power }\end{array}$ \\
\hline 19 & $\begin{array}{c}\text { Random, } \\
\text { Triangle, } \\
\text { Diamond, } \\
\text { Column }\end{array}$ & $\rho_{0}=-60 \mathrm{~dB}$ & $\sigma^{2}=-110 \mathrm{~dB}$ & 0.001 & $30 \mathrm{~mW}$ \\
\hline
\end{tabular}


The simulation results provide the network topology of the USVs' formation network, average delay, packet error ratio and connectivity ratio of each communication round. The network topology of four different USVs formation is shown in Fig. 6 and Fig. 7.
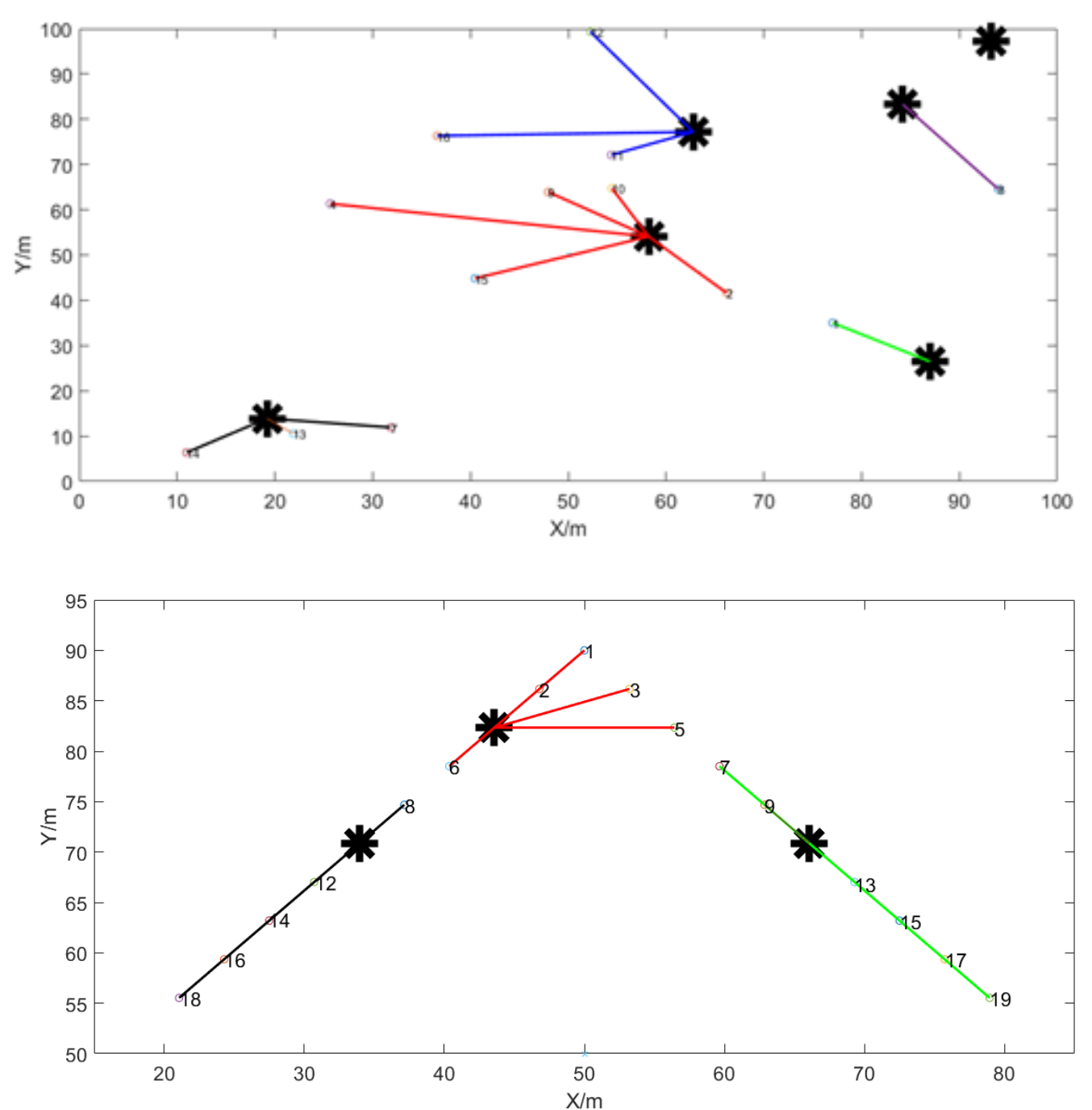

Fig. 6 Network topology diagram of random formation and triangle formation (The horizontal axis represents the $\mathrm{X}$ coordinate and the vertical axis represents $\mathrm{Y}$ coordinate. Nodes connected by same colour belong to one cluster, and the cluster head node is highlighted in black) 

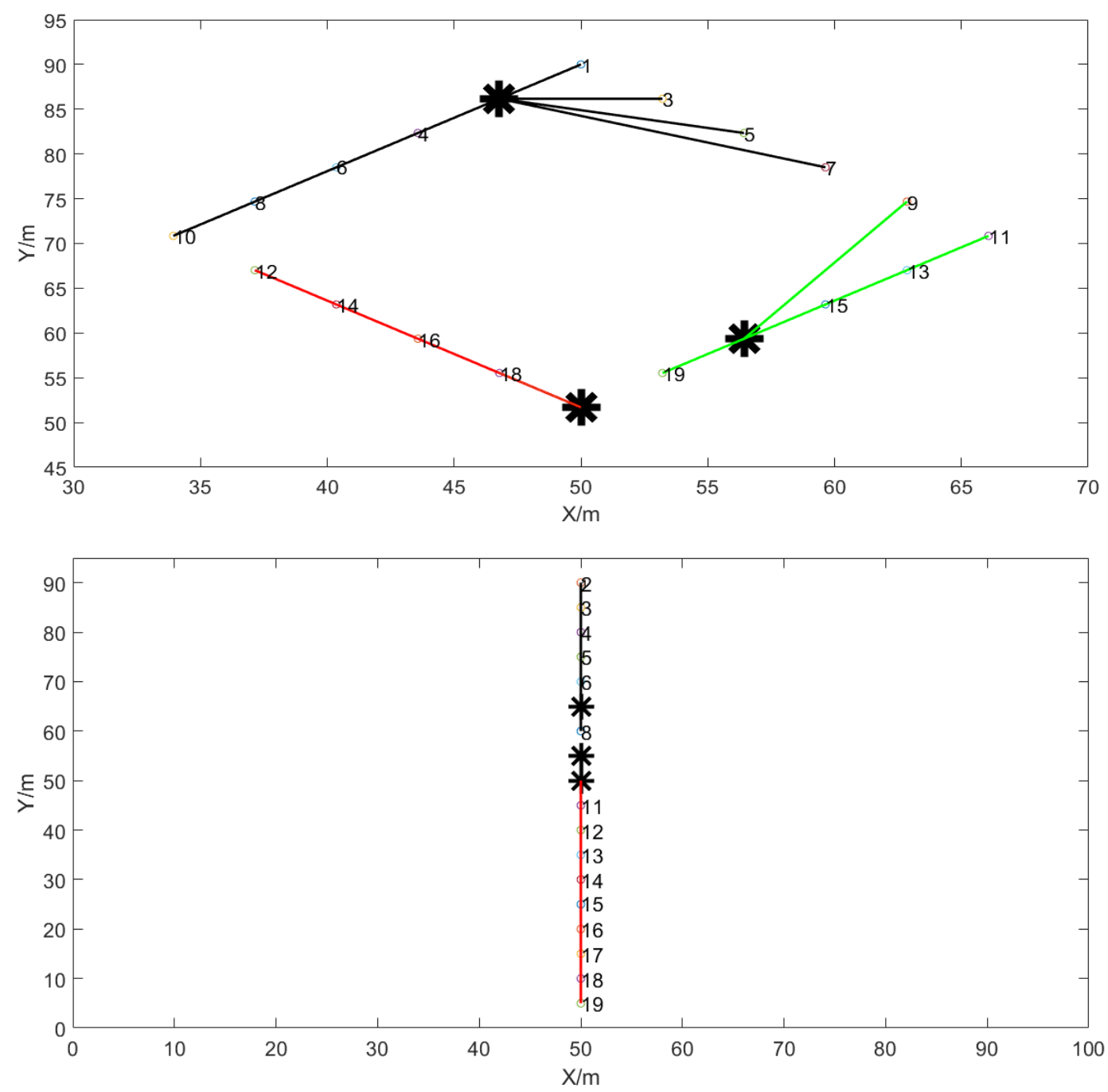

Fig. 7 Network topology diagram of diamond formation and column formation (The horizontal axis represents the $\mathrm{X}$ coordinate and the vertical axis represents $\mathrm{Y}$ coordinate. Nodes connected by same colour belong to one cluster, and the cluster head node is highlighted in black)

It can be seen in Fig. 6 and Fig. 7 cluster network topology is unlike the traditional network topology such as star, bus or circle structures. Cluster head node can organize normal nodes. Ordinary nodes are added to the nearest cluster head node through distance judgment according to the algorithm described in Fig. 4, thereby forming robust scalability and more flexible and controllable network topology. The simulated results related to the communication QoS indicators of different formations for packet error ratio, average time delay and connectivity are shown in Fig. 8, 9 and 10 respectively. 


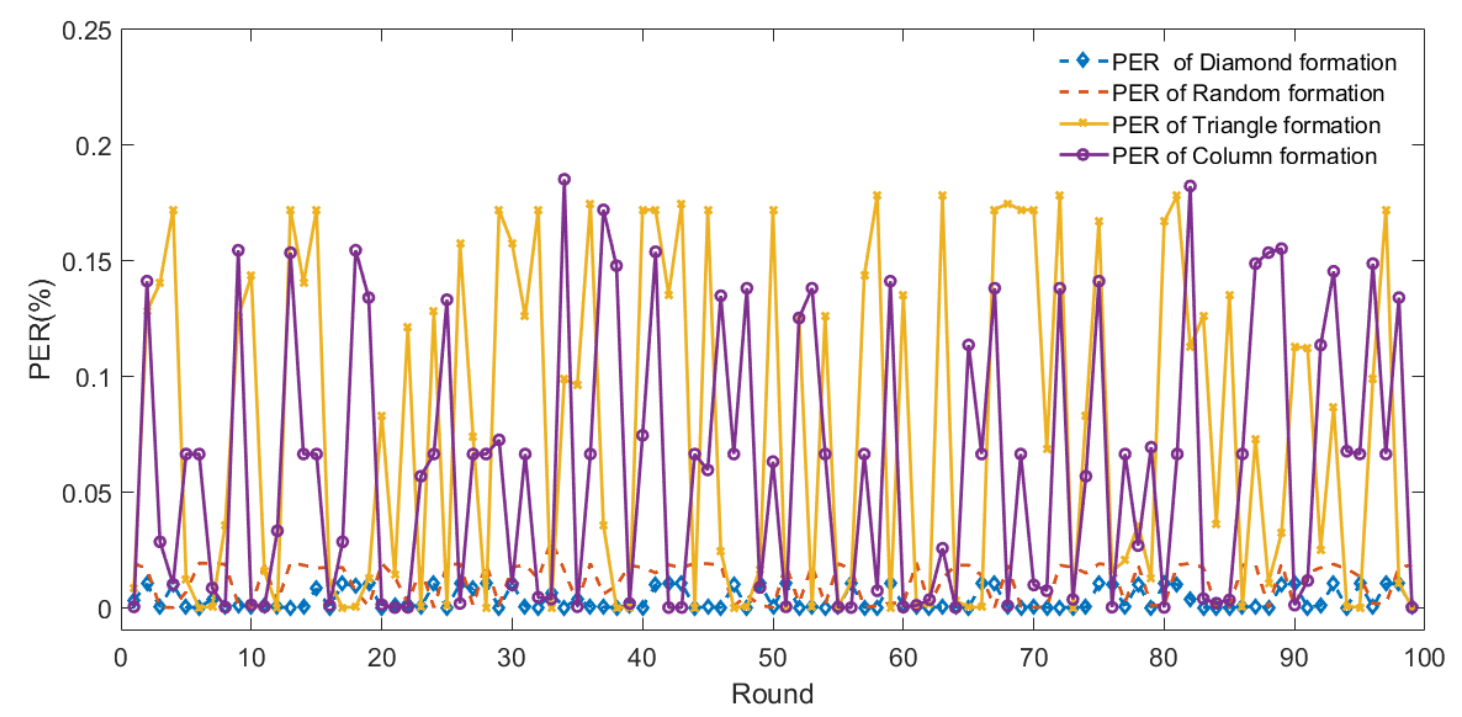

Fig. 8 Average packet error ratio curve under different formation shape

The average packet error ratio for four different communication networks topology of formation nodes i.e. diamond, triangle, column and random formation nodes, are depicted in Fig. 8. The same are distinguished with different colour coding. The horizontal axis represents the rounds of networking and the vertical axis represents the packet error ratio. The average packet error ratio of diamond formation nodes varies from $0.01 \%$ to $1.04 \%$, with an average of $0.35 \%$. The average packet error ratio of triangle formation nodes varies from $0.01 \%$ to $17.78 \%$, with an average value of $7.6 \%$. The average packet error ratio of the column formation nodes varies from $0.01 \%$ to $18.49 \%$, with an average value of $5.86 \%$. The average packet error ratio of random nodes varies from $0.02 \%$ to $2.88 \%$, with an average value of $1.13 \%$.

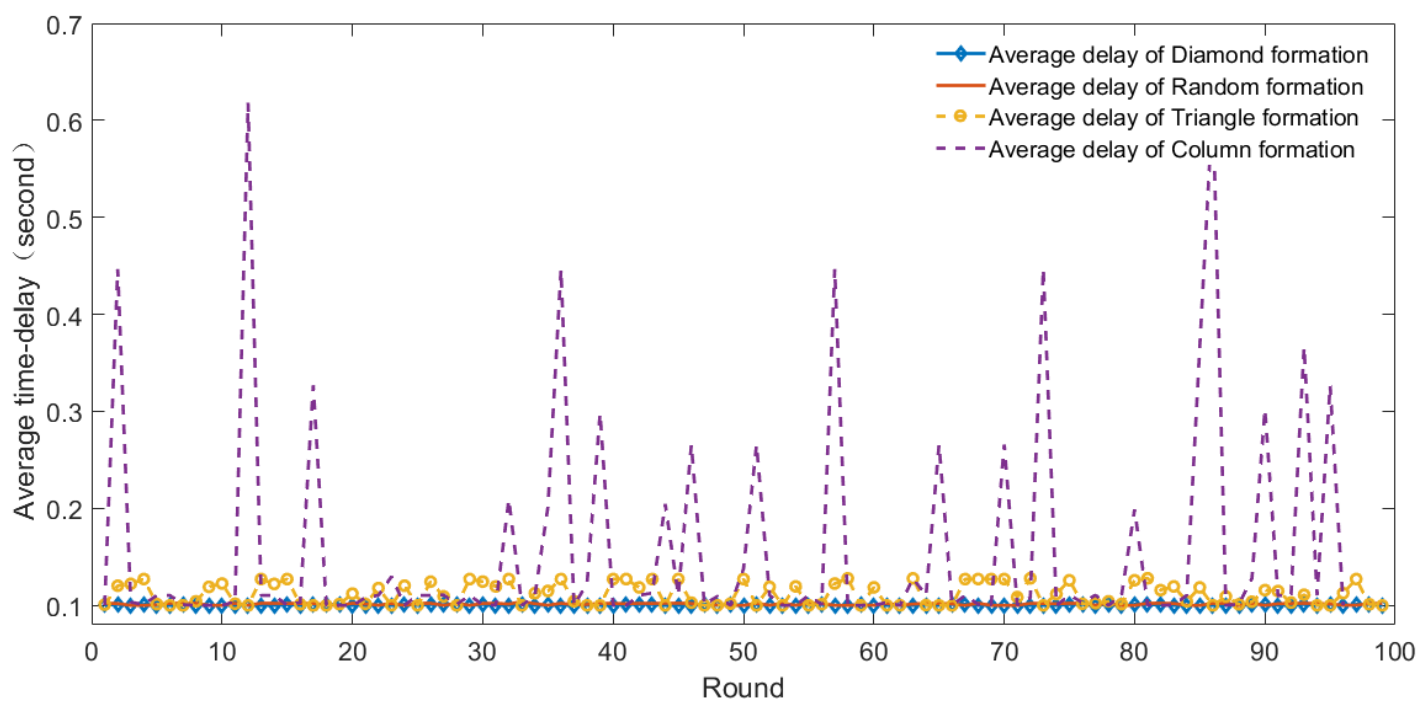

Fig. 9 Average time delay curve under different formation shape

The average delay variation curve for four different communication networks topology of formation nodes i.e. diamond, triangle, column and random formation nodes, are depicted in Fig. 9. The horizontal axis represents the rounds of networking and the vertical axis represents the average network delay. At the start of the vertical axis, 0.1 second represents the end-to-end time delay required to transmit certain control data between LOS link USV 
nodes without interference. The average network delay of the diamond formation node is 0.1 to $0.101 \mathrm{~s}$ with an average value of $0.100 \mathrm{~s}$. The average network delay of triangle formation nodes varies from 0.1 to $0.127 \mathrm{~s}$ with an average value of $0.111 \mathrm{~s}$. The average network delay of the column formation node varies from 0.1 to $0.618 \mathrm{~s}$ with an average value of $0.156 \mathrm{~s}$. The average network delay of random nodes varies from 0.1 to $0.103 \mathrm{~s}$ with an average value of $0.101 \mathrm{~s}$.

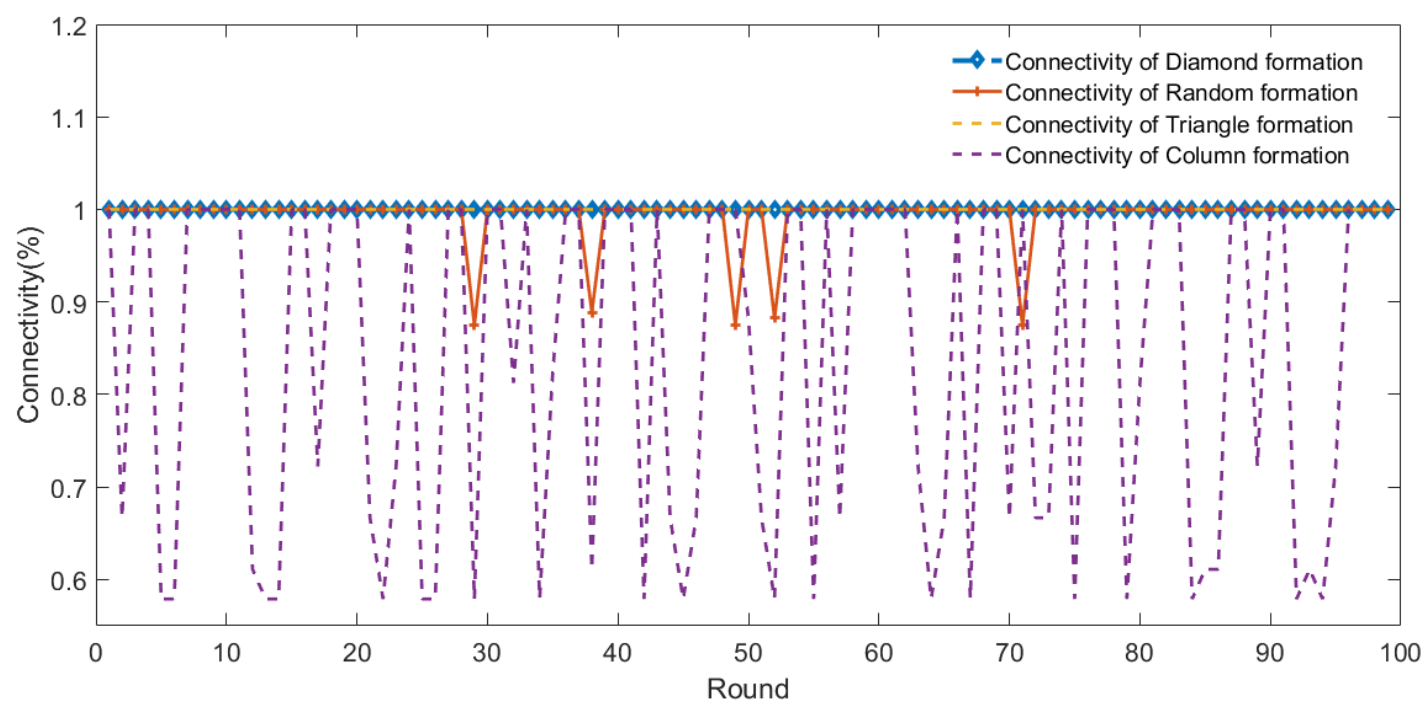

Fig. 10 Connectivity curve under different formation shape

The connectivity ratio of different formation structure is shown in Fig. 10. It can be seen connectivity ratio of diamond formation and column formation is $100 \%$. So, using the algorithm in Fig. 4 can guarantee diamond formation and column formation communication networks effectivity.

Simulation results reveal that different formation shapes have an impact on QoS indicators of the network. The packet error ratio and network delay of random formation nodes are lower than that of the triangle and column formation nodes. Triangle and column formation nodes are relatively scattered and the increase in distance affects the channel gain and thus not considered beneficial to QoS guarantee. In addition, random nodes sometimes appeared to be disconnected. QoS indicators such as time delay, packet error ratio depend on Signal to Interference and Noise Ratio. If USV nodes distance is too large, Signal will be weak. While, if USVs nodes distance is too close, Interference will be strong. Diamond shape is less dispersed than triangle, column shape. Hence the QoS parameters of diamond type formation are better. Consequently, stable geometric topologies such as diamond formation nodes are considered to be beneficial for guaranteed communication QoS. Therefore, the communication QoS indicator of USVs' networks under diamond formation shape is further investigated.

\subsubsection{QoS evaluation of diamond formation structure with different number of nodes}

In section 5.3.1, it shows that the diamond formation has better performance. Parameter settings for diamond formation are listed in Table 3. QoS indicators of diamond formation with 20, 28, 36, 44 nodes are evaluated. Packet error ratio, average time delay and connectivity curve are shown in Fig. 11, 12 and 13 respectively. 


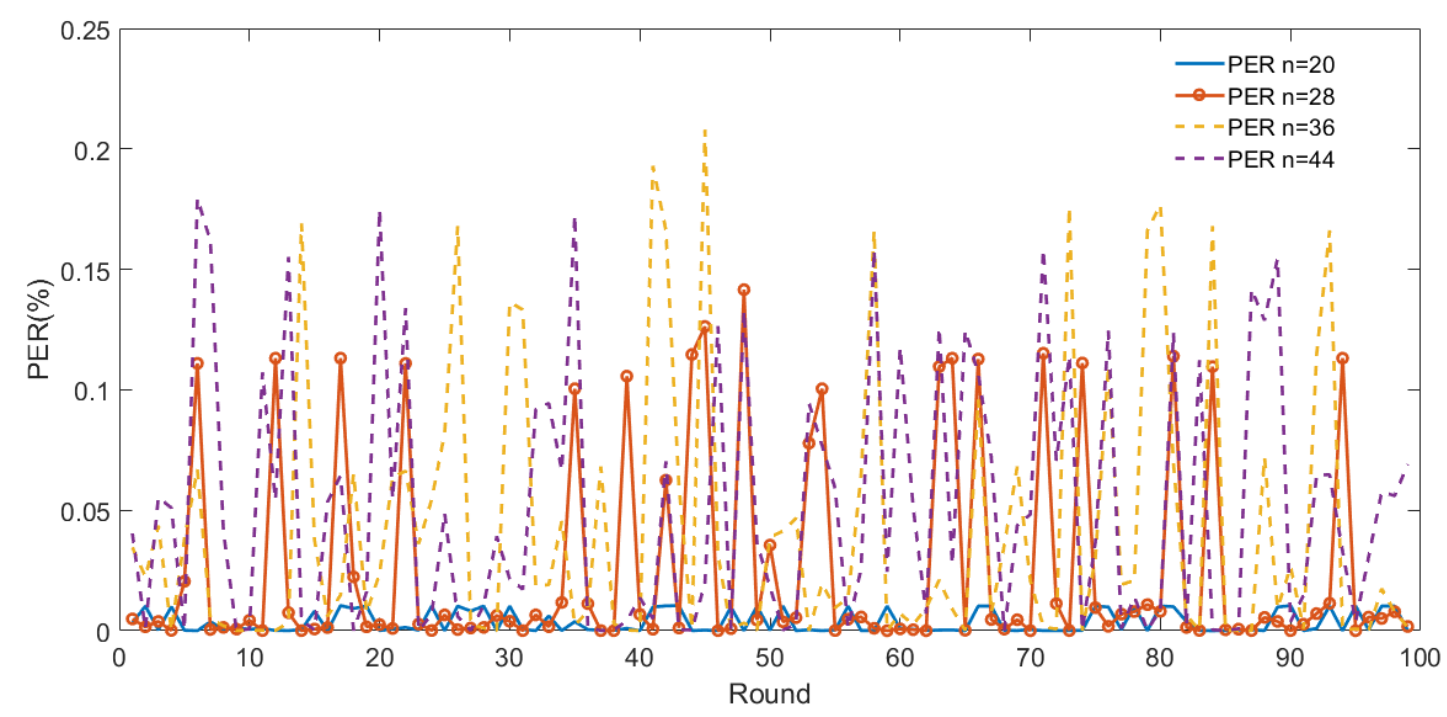

Fig. 11 Packet error ratio curve of diamond formation under different number of nodes

Fig. 11 depicts the change in the average packet error ratio for the communication round of USVs' formation network having number of nodes 20, 28, 36, and 44 with diamond shape. The horizontal axis represents the rounds of networking and the vertical axis represents the packet error ratio. It indicates that in the same formation configuration, the more nodes, the higher average packet error ratio. The details are as follows. When the number of nodes is 20, the average packet error ratio of the diamond formation nodes varies from $0.01 \%$ to $1.04 \%$ with an average value of $0.35 \%$. When the number of nodes is 28 , the average packet error ratio of diamond formation nodes varies from $0.01 \%$ to $14.15 \%$ with an average value of $2.49 \%$. When the number of nodes is 36 , the average packet error ratio of diamond formation nodes varies from $0.01 \%$ to $20.79 \%$ with an average value of $4.15 \%$. When the number of nodes is 44 , the average packet error ratio of the diamond formation nodes ranges from $0.02 \%$ to $17.94 \%$, with an average value of $5.15 \%$.

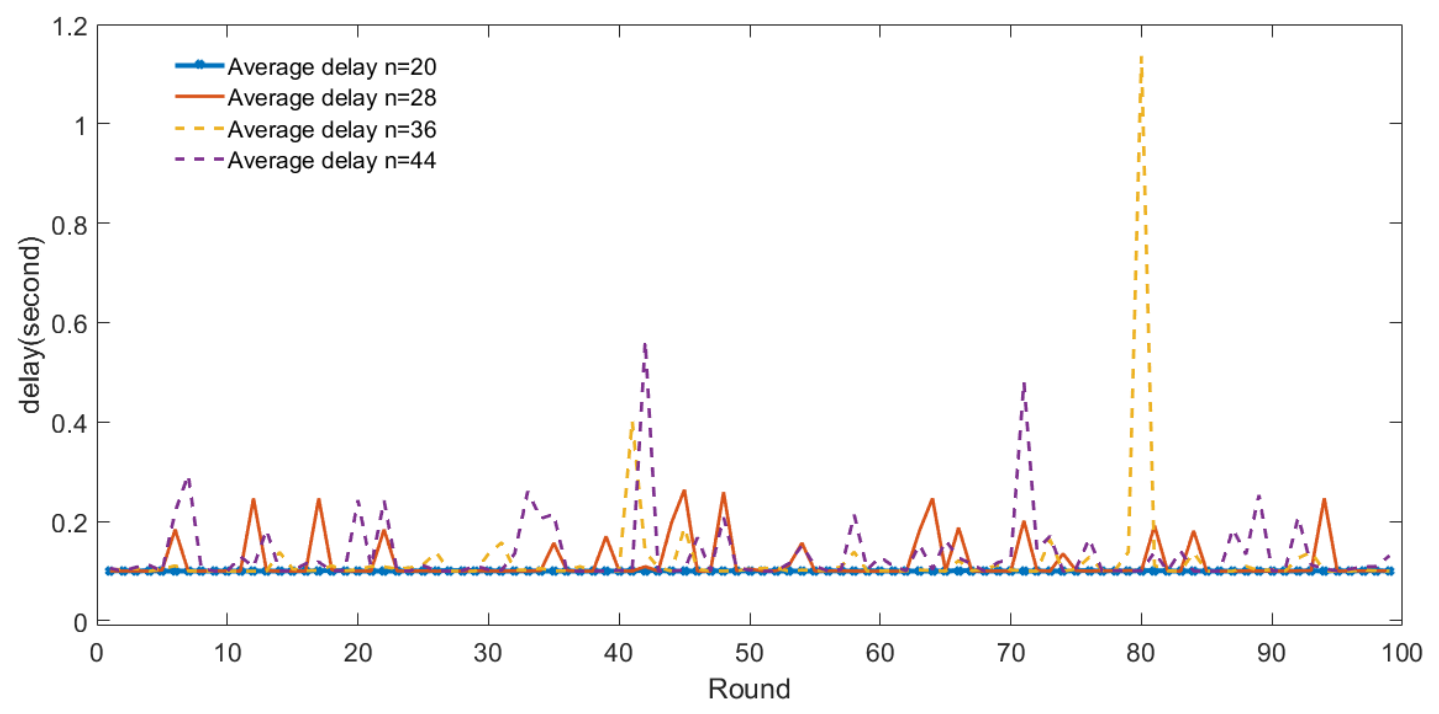

Fig. 12 Average delay curve of diamond formation under different number of nodes

Fig. 12 illustrates the change of the average network delay for the communication round of the USVs' formation network with number of nodes 20,28, 36, and 44 with diamond 
formation shape. The horizontal axis represents the rounds of networking and the vertical axis represents the average network delay.

It shows that in the same formation configuration, the more nodes, the higher average time delay. The details are as follows. When the number of nodes is 20 , the average delay of the diamond formation nodes varies from 0.1 to $0.101 \mathrm{~s}$ with an average value of $0.100 \mathrm{~s}$. When the number of nodes is 28 , the average delay of the diamond formation nodes varies from 0.1 to $0.263 \mathrm{~s}$ with an average value of $0.119 \mathrm{~s}$. When the number of nodes is 36 , the average delay of the diamond formation nodes varies from 0.1 to $1.136 \mathrm{~s}$ with an average value of $0.121 \mathrm{~s}$. While the node number is 44 , the average delay of the diamond formation nodes ranges from 0.1 to $0.562 \mathrm{~s}$ with an average value of $0.134 \mathrm{~s}$.

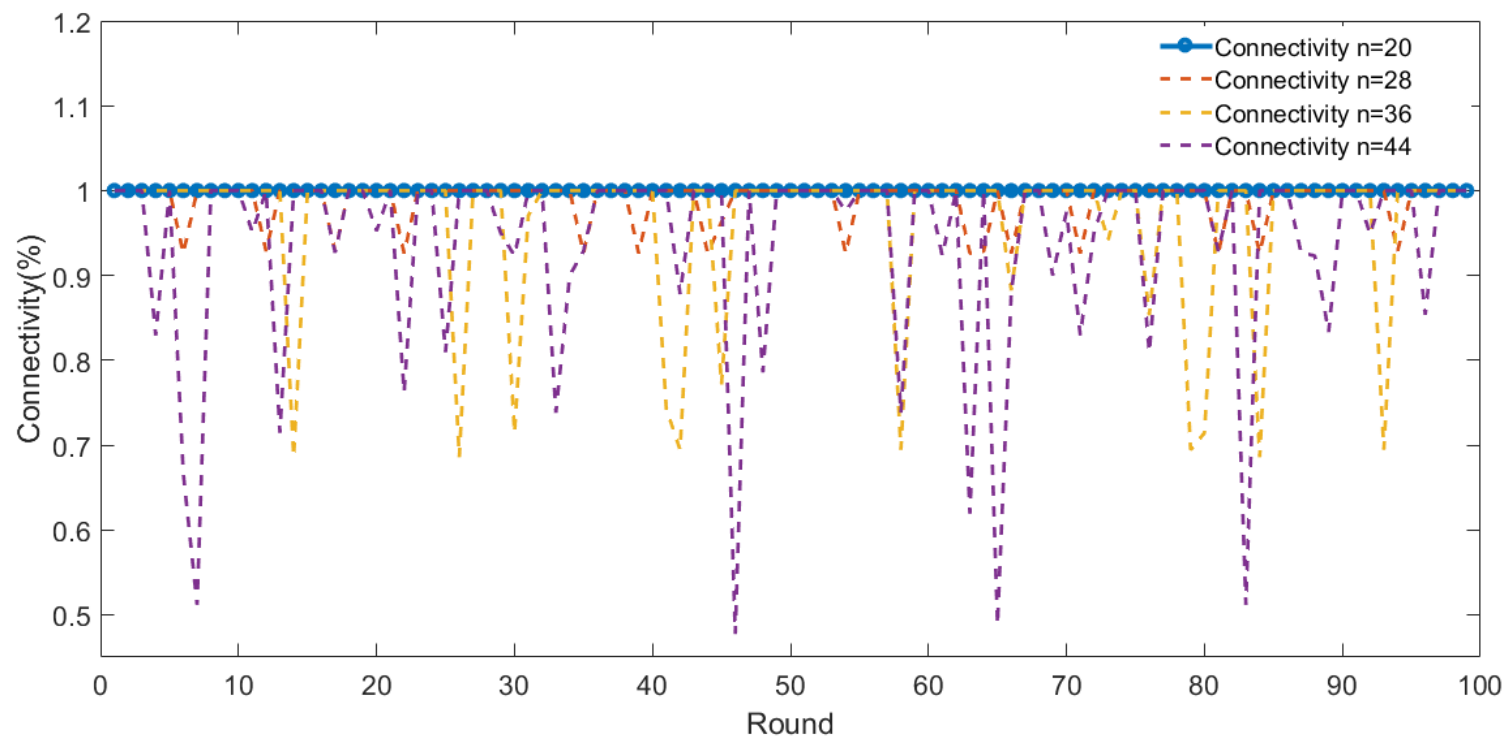

Fig. 13 Connectivity of diamond formation under different number of nodes

If the node number increases to certain value, the communication disconnection among USV nodes may occur under diamond formation as shown in Fig. 13. However, as the node number increases, the coverage of the surface operations can be improved based on the premise of network delay, packet error rate and other QoS indicators. Thus, evaluating QoS indicators of USVs formation can provide basic criterion for expanding the scale of USV formation and improving surface operation efficiency under the premise of ensuring communication demand.

\section{Conclusion}

In order to establish an efficient and reasonable communication networks for the formation control of multiple USVs, a hybrid communication networks architecture and communication networks algorithm are proposed. Moreover, QoS indicators are also considered in this paper, and QoS assessment method related to the communication model of USV fleet is presented. Numerical simulations to evaluate QoS indicators of USVs formation network are carried out. As the result, different formation shape of USV have different impacts on QoS indicators, and stable formation shape like diamond formation is beneficial to achieve the guaranteed QoS. With the increase of the node number, QoS indicators will be decreased. This can provide a criterion to select suitable number of USVs in formation to accomplish the mission in actual application scenario. Future work can be extended to the optimization problem between the geometric formation structure of the USV and the related QoS indicators. 


\section{ACKNOWLEDGEMENT}

This work was supported in part by the National Natural Science Foundation of China under Grant 52071153, in part by the Shenzhen Science and Technology Plan, China Project under Grant JCYJ201704I311305468, in part by the Fundamental Research Funds for the Central Universities, China under Grant 2018KFYYXJJ015 and Grant 2019JYCXJJ005.

\section{REFERENCES}

[1] Caoyang Yu, Xianbo Xiang, Lionel Lapierre, Qin Zhang. Robust magnetic tracking of subsea cable by AUV in the presence of sensor noise and ocean currents. IEEE Journal of Oceanic Engineering, 2018, 43(2): 311-322 https://doi.org/10.1109/JOE.2017.2768105

[2] Tatijana Dlabač, Martin Ćalasan, Maja Krčum, Nikola Marvučić. PSO-based PID controller design for ship course-keeping autopilot. Brodogradnja, 2019, 70(4): 1-15 https://doi.org/10.21278/brod70401

[3] Qin Zhang, Jialei Zhang, Ahmed Chemori, Xianbo Xiang. Virtual Submerged Floating Operational System for Robotic Manipulation. Complexity, 2018: 1-18. https://doi.org/10.1155/2018/9528313

[4] Francesco Mauro, Luca Braidotti, Giorgio Trincas. Determination of an optimal fleet for a CNG transportation scenario in the Mediterranean sea. Brodogradnja, 2019, 70(3): 1-23 https://doi.org/10.21278/brod70301

[5] JinJiang Li, Guandao Wang, Zhiheng Li, Shaolong Yang, Wentong Chong, Xianbo Xiang. A review on development of offshore wind energy conversion system. International Journal of Energy Research, 2020, 44(12): 9283-9297 https://doi.org/10.1002/er.5751

[6] Gong Xiang, Carlos Guedes Soares, Improved dynamical modelling of freely falling underwater cylinder based on cfd. Ocean Engineering 2020, 211:107538 https://doi.org/10.1016/j.oceaneng.2020.107538

[7] Nath Amar, Arun A. R., Niyogi Rajdeep. A distributed approach for road clearance with multi-robot in urban search and rescue environment. International journal of intelligent robotics and applications. 2019, 3(4): 392-406. https://doi.org/10.1007/s41315-019-00111-5

[8] Hongbo Wang, Jingxian Liu, Jinfen Zhang, Kezhong Liu, Xugang Yang, Qing Yu. Self-organizing cooperation model for ships navigating in restricted one-way waterway. Brodogradnja, 2018, 69(3): 1-23 https://doi.org/10.21278/brod69301

[9] Win Shane Kyi Hla,Win Luke Soe Thura, Soh Gim Song; et al. Design, modelling and control of collaborative samara autorotating wings (SAW). International journal of intelligent robotics and applications. 2019, 3(2): 144-157. https://doi.org/10.1007/s41315-019-00091-6

[10] Z. Peng, J. Wang, D. Wang and Q. Han. An Overview of Recent Advances in Coordinated Control of Multiple Autonomous Surface Vehicles. IEEE Transactions on Industrial Informatics, 2020, https://doi.org/10.1109/TII.2020.3004343

[11] M. Mike, A. Erkki, V. Reino, et al. Using OPNET to Model and Evaluate the MU Performance based on IEC 61850-9-2LE. Procedia Computer Science, 2014, 36:72-79. https://doi.org/10.1016/j.procs.2014.09.040

[12] H. Nawaz, H. Ali, A. Laghari. UAV Communication Networks Issues: A Review. Archives of Computational Methods in Engineering (2020). http://dx.doi.org/10.1007/s11831-020-09418-0.

[13] V. Sharma, R. Kumar. A Cooperative Network Framework for Multi-UAV Guided Ground Ad Hoc Networks. Journal of Intelligent \& Robotic Systems, 2015, 77:629-652. https://doi.org/10.1007/s10846014-0091-0

[14] T. Zhu, S. Dhelim, Z. Zhou, et al. An Architecture for Aggregating Information from Distributed Data Nodes for Industrial Internet of Things. Computers \& Electrical Engineering (2016). http://dx.doi.org/10.1016/j.compeleceng.2016.08.018

[15] T. Qiu, Y. Lv, F, et al. ERGID: An Efficient Routing Protocol for Emergency Response Internet of Things. Journal of Network and Computer Applications 72 (2016): 104-12. https://doi.org/10.1016/j.jnca.2016.06.009

[16] T. Qiu, D. Luo, F. Xia, et al. A Greedy Model with Small World for Improving the Robustness of Heterogeneous Internet of Things. Computer Networks, 2016, 101:127-143. https://doi.org/10.1016/j.comnet.2015.12.019

[17] Pham V T, Messai N, Nguyen D H, et al. Robust formation control under state constraints of multi-agent systems in clustered networks. Journal of Systems \& Control Letters, 2020, 140:104689. https://doi.org/10.1016/j.sysconle.2020.104689 
[18] Zhao Wang, Shaolong Yang, Xianbo Xiang, Antonio Vasilijević, Nikola Mišković, Đula Nad. Cloudbased mission control of USV fleet: architecture, implementation and experiments. Control Engineering Practice, 2020, https://doi.org/10.1016/j.conengprac.2020.104657

[19] P. Li, Z. Liu, C. He, et al. Distributed adaptive fault-tolerant control for spacecraft formation with communication delays. IEEE Access (2020). http://dx.doi.org/10.1109/ACCESS.2020.3003681.

[20] H. Namgung, J. Jeong, J. Choi. An Experimental Result on Information Exchange using USV Communication Relay System. Physics: Conference Series (2019). http://dx.doi.org/10.1088/17426596/1357/1/012043.

[21] K. Gill, S. Yang, F. Yao, et al. A ZigBee-Based Home Automation System. IEEE Transactions on Consumer Electronics, 2009, 55(2):422-430. https://doi.org/10.1109/TCE.2009.5174403

[22] M. P. Praveen, M. R. Babu. Energy Efficient Cluster Head Selection for Internet of Things. New Review of Information Networking, 2017, 22(1):54-70. https://doi.org/10.1080/13614576.2017.1297734

[23] Cui Z, Cao Y, Cai X, et al. Optimal LEACH protocol with modified bat algorithm for big data sensing systems in Internet of Things. Parallel and Distributed Computing, 2019, 132:217-229. https://doi.org/10.1016/j.jpdc.2017.12.014

[24] Dixon C, Frew E W. Optimizing Cascaded Chains of Unmanned Aircraft Acting as Communication Relays. IEEE Journal on Selected Areas in Communications, 2012, 30(5):883-898. ttps://doi.org/10.1109/JSAC.2012.120605

[25] Liu Q, Zhou S, Giannakis G B. Cross-Layer combining of adaptive Modulation and coding with truncated ARQ over wireless links. IEEE Transactions on Wireless Communications, 2004, 3(5): 1746-1755. https://doi.org/10.1109/TWC.2004.833474

[26] L. Bandyopadhyay, S. Chaulya, et al. Line-of-Sight Communication. Wireless Communication in Underground Mines, 2010:105-120. https://doi.org/10.1007/978-0-387-98165-9_5.

Submitted: 21.08.2020.

Accepted:
Shaoze Zhang

School of Naval Architecture and Ocean Engineering,

Huazhong University of Science and Technology 430074, Wuhan, China

Xianbo Xiang

School of Naval Architecture and Ocean Engineering,

Huazhong University of Science and Technology 430074, Wuhan, China

Shenzhen Huazhong University of Science and Technology Research Institute, Shenzhen 518057, China

Hubei Key Laboratory of Naval Architecture and Ocean Engineering Hydrodynamics (HUST), Wuhan, China

Shaolong Yang* , Corresponding author: yangsl@hust.edu.cn

School of Naval Architecture and Ocean Engineering,

Huazhong University of Science and Technology 430074, Wuhan, China

Hubei Key Laboratory of Naval Architecture and Ocean Engineering Hydrodynamics (HUST), Wuhan, China

Collaborative Innovation Center for Advanced Ship and Deep-Sea Exploration (CISSE), Shanghai, China 